\title{
An experiment of nature: genetic L-arginine deficiency and NO insufficiency
}

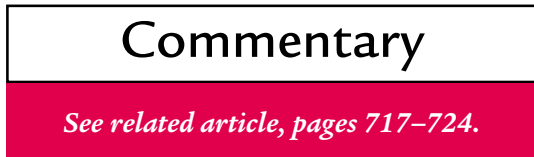

\begin{abstract}
Joseph Loscalzo
Whitaker Cardiovascular Institute and Evans Department of Medicine, Boston University School of Medicine, 700 Albany Street, W507, Boston, Massachusetts 02118, USA. Phone: (617) 638-4890; Fax: (617) 638-4066; E-mail: jloscalz@bu.edu.

J. Clin. Invest. 108:663-664 (2001). DOI:10.1172/JCI200113848.
\end{abstract}

Nitric oxide $(\mathrm{NO})$ is derived from the oxidation of the semiessential amino acid L-arginine by the NO synthases. Evidence supporting a central role for $\mathrm{L}$-arginine in the NO synthase pathway comes from several observations. First, NO synthesis by cultured endothelial cells depends upon L-arginine content of the media (1). In addition, NO synthesis is blocked by L-arginine analogues (2), such as $\mathrm{N}^{\mathrm{G}}$-monomethyl-Larginine. Asymmetric dimethylarginine (ADMA), an endogenous compound known to inhibit NO synthases (3), and L-glutamine, which inhibits the generation of L-arginine from L-citrulline by argininosuccinate synthetase (4), also impair endothelial NO synthesis (5). Conversely, supplemental L-arginine improves endothelial function (6) and reverses endothelial dysfunction (7).

In this issue of the JCI, Kamada and colleagues (8) report the results of vascular studies of an individual with a rare genetic disorder of L-arginine metabolism that lends further support to the critical role of L-arginine in $\mathrm{NO}$ synthesis. The authors describe a patient with a rare autosomal recessive disorder, lysinuric protein intolerance, characterized by a defect in dibasic amino acid transport caused by mutations in the SLC7A7 gene. The subject tion in the patient is considerably lower than normal (reduced by $79 \%$ ).

In view of the effect of this genetic experiment of nature on plasma L-arginine concentrations and the importance of $\mathrm{L}$-arginine on vascular NO production, Kamada et al. assessed NO-dependent endothelial function in this patient before and after intravenous L-arginine supplementation. They found that his serum level of nitrogen oxides $\left(\mathrm{NO}_{\mathrm{x}}\right)$ and his flow-mediated brachial artery vasodilator response were each approximately $70 \%$ lower than in controls. Intravenous infusion of L-arginine caused an increase in $\mathrm{NO}_{x}$ levels and restored normal flow-mediated vasodilator responses in this patient, whose coronary arteriogram was normal. Exercise treadmill testing and positron emission tomography (using $\left[{ }^{13} \mathrm{~N}\right]$-ammonia) of the heart both showed evidence of myocardial ischemia, which could be ameliorated by intravenous administration of L-arginine. In addition to these abnormalities in vasomotor function, an earlier report showed that this patient also had a reduced circulating platelet count, increased plasma levels of the thrombin-antithrombin III complex, and elevated plasma fibrin(ogen)

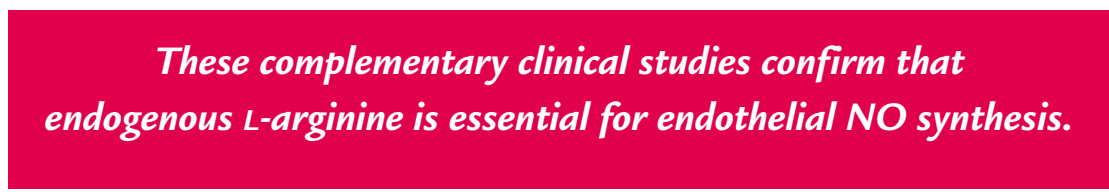

is a compound heterozygote carrying two different putative null alleles. The transporter is normally expressed in intestinal and renal epithelial cells, and deficient expression leads to impaired dietary uptake of exogenous $\mathrm{L}$-arginine and impaired renal tubular reabsorption of filtered L-arginine. As a result, plasma L-arginine concentra- degradation products. Importantly, intravenous L-arginine increased the platelet count and reduced the levels of thrombin-antithrombin III complex and fibrin(ogen) degradation products (9), as well.

Taken together, these complementary clinical studies confirm that endogenous L-arginine is essential for endothe- lial NO synthesis and demonstrate that a naturally occurring deficiency of endothelial NO production is associated with endothelial dysfunction, manifest as abnormal vasomotor tone and a prothrombotic state. Significantly, replenishing the substrate by intravenous administration of $\mathrm{L}$-arginine restores normal endothelial function. These data support the view that endothelial NO derived from L-arginine maintains tissue perfusion by its vasodilator and antithrombotic effects.

\section{Puzzles remaining}

Several questions derive from these simple observations. First, why should a reduction of the plasma concentration of L-arginine to this level lead to reduced endothelial NO synthase (eNOS) activity and NO production? The intracellular concentration of L-arginine ranges from $100 \mu \mathrm{M}$ to 2 $\mathrm{mM}$ in freshly isolated endothelial cells (7), implying the existence of an extracellular-to-intracellular concentration gradient against which the L-arginine $\left(y^{+}\right)$transporters drive $\mathrm{L}$-arginine into cells. Whether the reduced extracellular concentration of L-arginine in this patient leads to less efficient cellular uptake is not known. Other factors that influence the rate of L-arginine transport into endothelial cells, in addition to L-arginine concentration, include membrane potential, $\mathrm{K}^{+}$concentration, other cationic amino acids (especially lysine and glutamine), and glucose and insulin concentrations (10). Most investigators believe that because the high-affinity cationic arginine transporter (CAT-1) colocalizes in caveolae with membrane-bound eNOS (11), extracellular L-arginine concentration is the most important determinant of L-arginine availability for eNOS. Since the plasma and assumed intracellular concentrations of L-arginine in this patient still far exceed the $K_{\mathrm{M}}$ for eNOS $(2.9 \mu \mathrm{M}$ for purified 
eNOS [ref. 12]), there should be no substrate limitation for enzyme activity. Nevertheless, L-arginine infusion clearly increases endothelial NO production in this patient, suggesting that the in vitro kinetic constants do not apply in vivo, either because of compartmentalization of substrate and enzyme (in caveolae, for example), the presence of other uncharacterized factors that modulate enzyme function or substrate availability, or both.

Second, why is the patient not hypertensive? The authors speculate that there must be other compensatory vasodilators that offset the loss of bioactive NO in this patient, thus maintaining his blood pressure in the normal range. Measurement of circulating concentrations of prostaglandins and other endogenous vasodilators would be needed to substantiate this hypothesis. Another possible explanation is that at lower concentrations of L-arginine, the concentration of the naturally occurring competitive inhibitor of eNOS, ADMA (5), is correlatively reduced perhaps below its $K_{\mathrm{I}}$ - leading to a greater effective concentration of L-arginine for the enzyme. Without measurements of the $K_{\mathrm{I}}$ for ADMA and of the intra- and extracellular concentrations of ADMA in this patient, this mechanism also remains speculative.

Third, did this patient have evidence for increased oxidant stress? In the absence of sufficient substrate, eNOS changes its enzymatic profile: Instead of oxidizing L-arginine to L-citrulline and $\mathrm{NO}$, it reduces molecular oxygen to superoxide anion, a process termed enzymatic "uncoupling." Again, without some measure of reactive oxygen species (e.g., $\mathrm{F}_{2}$-isoprostanes), this question also remains unanswered.

Fourth, why did the patient show signs of ongoing consumptive coagu- lopathy? If he made sufficient NO to prevent hypertension, why was this level of NO insufficient to impair thrombosis? Obviously, whatever the flux of NO in this patient, it was inadequate to limit thrombus formation. A growing body of data supports the greater importance of endothelial NO as an inhibitor of platelet function and thrombosis than as a vasodilator (for review, see ref. 13). The endothelium is not the only source of vascular NO that serves an antithrombotic purpose: platelet-derived NO limits recruitment of other platelets to the growing thrombus (14). In addition, L-arginine suppresses tissue factor expression by cultured endothelial cells (15), suggesting that the exogenous amino acid reduced the prothrombotic state in this patient both by promoting the production of endothelial- and platelet-derived NO and by inhibiting tissue factor activity. Measures of endothelial- or plateletderived $\mathrm{NO}$ before and after $\mathrm{L}$-arginine infusion would be needed to test these possible explanations.

Clearly, straightforward vascular and hemostatic studies of this single patient have provided important insights into the in vivo role of the L-arginine-NO axis in vascular homeostasis. The derivative questions posed here reflect the very interesting nature of this complex system. Well-done studies invariably lead to more questions: simply taking NO for an answer to these questions has its limitations.

1. Palmer, R.M.J., Rees, D.D., Ashton, D.S., and Moncada, S. 1988. L-arginine is the physiological precursor for the formation of nitric oxide in endothelium-dependent relaxation. Biochem. Biophys. Res. Comm. 153:1251-1256.

2. Gross, S.S., et al. 1990. Macrophage and endothelial nitric oxide synthesis: cell-type selective inhibition by $\mathrm{N}^{\mathrm{G}}$-aminoarginine, $\mathrm{N}^{\mathrm{G}}$ nitroarginine, and $\mathrm{N}^{\mathrm{G}}$-methylarginine. Biochem.
Biophys. Res. Comm. 150:96-103.

3. Vallance, P., Leone, A., Calver, A., Collier, J., and Moncada, S. 1992. Accumulation of an endogenous inhibitor of nitric oxide synthesis in chronic renal failure. Lancet. 339:572-575.

4. Sessa, W.C., Hecker, M., Mitchell, J.A., and Vane J.R. 1990. The metabolism of L-arginine and its significance for the biosynthesis of endothelium-derived relaxing factor: L-glutamine inhibits the generation of L-arginine by cultured endothelial cells. Proc. Natl. Acad. Sci. USA. 87:8607-8611

5. Hecker, M., Sessa, W.C., Harris, H.J., Anggard, E.E., and Vane, J.R. 1990. The metabolism of Larginine and its significance for the biosynthesis of endothelium-derived relaxing factor: cultured endothelial cells recycle L-citrulline to L-arginine. Proc. Natl. Acad. Sci. USA 87:8612-8616.

6. Chin-Dusting, J.P.F., et al. 1996. Effects of in vivo and in vitro L-arginine supplementation on healthy human vessels. J. Cardiovasc. Pharm. 28:158-166

7. Creager, M.A., Gallagher, S.M., Girerd, X.J. Dzau, V.J., and Cooke, J.P. 1992. L-Arginine improves endothelium-dependent vasodilation in hypercholesterolemic humans. J. Clin. Invest. 90:1248-1253

8. Kamada, Y., et al. 2001. Vascular endothelial dysfunction resulting from L-arginine deficiency in a patient with lysinuric protein intolerance. $J$. Clin. Invest. 108:717-724.

9. Kayanoki, Y., et al. 1999. Reduced nitric oxide production by L-arginine deficiency in lysinuric protein intolerance exacerbates intravascular coagulation. Metabolism. 48:1136-1140.

10. Zharikov, S.I., and Block, E.R. 1998. Characterization of L-arginine uptake by plasma membrane vesicles isolated from cultured pulmonary artery endothelial cells. Biochim. Biophys. Acta. 1369:173-183.

11. McDonald, K.K., Zharikov, S., Block, E.R., and Kilberg, M.S. 1997. A calveolar complex between the cationic amino acid transporter I and endothelial nitric-oxide synthase may explain the "arginine paradox." J. Biol. Chem. 272:31213-31216.

12. Pollock, J.S., et al. 1991. Purification and characterization of particulate endothelium-derived relaxing factor synthase from cultured and native bovine aortic endothelial cells. Proc. Natl. Acad. Sci. USA. 88:10480-10484.

13. Loscalzo, J. 2001. Nitric oxide insufficiency, platelet activation, and arterial thrombosis. Circ Res. 88:756-762.

14. Freedman, J.E., et al. 1997. Nitric oxide released from activated platelets inhibits platelet recruitment. J. Clin. Invest. 100:350-356.

15. Yang, Y, and Loscalzo, J. 2000. Regulation of tissue factor expression in human microvascular endothelial cells by nitric oxide. Circulation 101:2144-2148. 